\title{
Efeito da alimentação de Schizaphis graminum com genótipos de sorgo no desenvolvimento do predador Cycloneda sanguinea ${ }^{(1)}$
}

\author{
Terezinha Monteiro dos Santos ${ }^{(2)}$, Liliane Karla Figueira(2), Arlindo Leal Boiça Júnior(2), \\ Fernando Mesquita Lara ${ }^{(2)}$ e Ivan Cruz $^{(3)}$
}

\begin{abstract}
Resumo - O pulgão Schizaphis graminum (Rondani) (Hemiptera: Aphididae) é uma das principais pragas do sorgo. O objetivo deste trabalho foi determinar o efeito da alimentação deste inseto com genótipos de sorgo resistentes e suscetíveis no desenvolvimento do predador Cycloneda sanguinea (Linnaeus) (Coleoptera: Coccinellidae). O pulgão foi criado em folhas dos genótipos de sorgo GR 11111 e TX 430 x GR 111, resistentes, GB 3B, de resistência moderada e BR 007B, suscetível ao hemíptero. As larvas de C. sanguinea foram alimentadas diariamente, ad libitum, com ninfas e adultos de $S$. graminum. A duração das fases de desenvolvimento e a sobrevivência de $C$. sanguinea não foram influenciadas pelo genótipo. Apenas a duração do quarto ínstar, da fase larval e do período de larva a adulto foram afetadas. O peso de larvas de segundo ínstar de $C$. sanguinea não foi afetado pelo genótipo. Nos demais ínstares e fase adulta, esse parâmetro foi diferenciado conforme o genótipo de sorgo utilizado como hospedeiro ao pulgão. Os genótipos resistentes GR 11111 e TX 430 x GR 111 não afetam adversamente o desenvolvimento e a fecundidade de C. sanguinea, durante uma geração. Estes genótipos demonstram compatibilidade com o predador e viabilizam o manejo de $S$. graminum na cultura do sorgo.
\end{abstract}

Termos para indexação: larva, etapa de desenvolvimento, planta hospedeira, resistência às pragas, controle biológico.

\section{Effect of Schizaphis graminum feeding on sorghum genotypes on the development} of its predator Cycloneda sanguinea

\begin{abstract}
The aphid Schizaphis graminum (Rondani) (Hemiptera: Aphididae) is one of the main pests of sorghum crop. The objective of this work was to evaluate the effect of this aphid feeding on resistant and susceptible sorghum genotypes on the development of its predator Cycloneda sanguinea (Linnaeus) (Coleoptera: Coccinellidae). The aphid was reared on leaves of sorghum genotypes GR 11111 and TX $430 \mathrm{x}$ GR 111, resistant, GB 3B, moderately resistant, and BR 007B, susceptible to the hemipterous. Larvae of C. sanguinea were fed ad libitum on S. graminum nymphs and adults, daily. The duration and survival of developmental phases of $C$. sanguinea were not significantly influenced by genotype. Only the fourth instar, the larval phase and the larvae-adult period duration were affected. The weight of $C$. sanguinea larvae of second instar was not affected by sorghum genotype. For both instars and adult phase, this parameter was differentiate due to each genotype used as host to the aphid. The resistant genotypes GR 11111 and TX 430 x GR 111 do not affect adversely $C$. sanguinea development and fecundity, during one generation. These genotypes show compatibility with the predator, allowing the $S$. graminum management on sorghum crop.
\end{abstract}

Index terms: larvae, developmental stages, host plants, pest resistance, biological control.

(1) Aceito para publicação em 20 de fevereiro de 2003.

(2) Universidade Estadual Paulista, Fac. de Ciências Agrárias e Veterinárias, Dep. de Fitossanidade, Via de Acesso Prof Paulo Donato Castellane, s/n, CEP 14884-900 Jaboticabal, SP. E-mail: tmonteirosantos@ig.com.br, lkfigueira@yahoo.com.br, aboicajr@fcav.unesp.br,fmlara@fcav.unesp.br

(3) Embrapa-Centro Nacional de Pesquisa de Milho e Sorgo, Caixa Postal 151, CEP 35701-970 Sete Lagoas, MG

E-mail: ivancruz@cnpms.embrapa.br

\section{Introdução}

Entre os inimigos naturais, os coccinelídeos são os predadores mais relacionados com o controle biológico de insetos-praga (Obrycki \& Kring, 1998). Aproximadamente 490 gêneros e 4.200 espécies têm sido descritos e, entre estas espécies, $90 \%$ são consideradas benéficas, principalmente por sua ação pre- 
dadora contra pulgões, cochonilhas, moscas-brancas e ácaros (Iperti, 1999).

Muitos fatores em um agroecossistema, como plantas hospedeiras das pragas, podem afetar a colonização, desenvolvimento e eficiência de inimigos naturais que exercem o controle biológico (Botrell et al., 1998). As plantas podem influenciar direta ou indiretamente a eficiência dos coccinelídeos, alterando a qualidade da presa ou as chances de encontro desta pelo predador (Rice \& Wilde, 1989).

Programas de melhoramento genético têm incorporado características de resistência em plantas, sem considerar como esta resistência ou se outras características da planta podem afetar os inimigos naturais (Botrell et al., 1998). Interações antagonistas entre a resistência de plantas e controle biológico são causadas freqüentemente por substâncias químicas secundárias da planta (Verkerk et al., 1998); os efeitos prejudiciais da resistência do tipo antibiose são passados do inseto fitófago ao seu predador (Farid et al., 1997). Larvas de Hippodamia convergens Guérin-Méneville alimentadas com o pulgão Schizaphis graminum (Rondani), criado em cultivares de sorgo com resistência do tipo antibiose, apresentaram menor viabilidade larval e pupal e maior período de desenvolvimento (Rice \& Wilde, 1989).

Ao contrário, pesquisas na mesma linha têm demonstrado a compatibilidade entre cultivares resistentes e controle biológico. Plantas resistentes, principalmente com efeitos de antibiose, podem alterar o crescimento populacional de pulgões, reduzindo a atividade e alimentação destes hemípteros, aumentando a razão predador/presa e evitando que a população do fitófago alcance densidades na qual o inimigo natural torne-se ineficiente (Messina \& Sorenson, 2001).

Os períodos larval, pré-pupal, pupal e de larva-adulta do coccinelídeo Scymnus frontalis (Fabricius) não foram diferentes quando este predador alimentou-se de pulgões Diuraphis noxia (Mordvilko), criados em cultivares de trigo suscetíveis e resistentes (Farid et al., 1997). A capacidade predatória de pulgões Myzus persicae (Sulzer) por adultos de Cycloneda sanguinea (Linnaeus) não foi influenciada por genótipos de batata (Silva \& Lara, 1999). Os efeitos de cultivares resistentes de trigo e a predação do pulgão D. noxia por Chrysoperla plorabunda (Fitch) foram sinergísticos. As larvas do crisopídeo causaram maior redução na densidade populacional do pulgão em cultivares tolerantes do que nas suscetíveis (Messina \& Sorenson, 2001). De acordo com Giles et al. (2002), larvas de $H$. convergens sobreviveram e se desenvolveram melhor quando foram supridas com pulgões Acyrthosiphon kondoi Shinji criados em cultivares de alfafa resistentes. Associações positivas foram observadas por Figueira et al. (2002) entre o predador Chrysoperla externa (Hagen), e genótipos de sorgo resistente e moderadamente resistente ao pulgão $S$. graminum, evidenciando a possibilidade da integração resistência de plantas e controle biológico.

$O$ pulgão $S$. graminum é uma das principais pragas da cultura do sorgo e o uso de variedades resistentes tem sido um dos métodos mais eficazes em seu controle (Cruz \& Vendramim, 1998). Na cultura do sorgo, os genótipos GR, TX 430 x GR e GB 3 apresentam o tipo de resistência não-preferência (Cruz \& Vendramim, 1989) e antibiose a S. graminum (Cruz \& Vendramim, 1995). Uma planta com resistência do tipo antibiose provoca um efeito adverso sobre a biologia da praga ao passo que a cultivar com resistência do tipo não-preferência é menos utilizada pelo inseto para alimentação, oviposição ou abrigo (Lara, 1991). Além da resistência, o pulgão-verde do sorgo é também controlado por seus inimigos naturais, destacando-se entre estes os coccinelídeos, principalmente a espécie $C$. sanguinea. Isenhour et al. (1989) sugerem a combinação de plantas resistentes e inimigos naturais no controle de insetos-praga, mas enfatizando a necessidade de pesquisas prévias sobre a compatibilidade de genótipos com os agentes bióticos envolvidos.

O objetivo deste trabalho foi determinar o efeito de genótipos de sorgo, utilizados para alimentar o pulgão Schizaphis graminum, no desenvolvimento do predador Cycloneda sanguinea.

\section{Material e Métodos}

O trabalho foi realizado no Departamento de Fitossanidade, Universidade Estadual Paulista (Unesp), Faculdade de Ciências Agrárias e Veterinárias (FCAV), Campus de Jaboticabal, SP. Utilizaram-se os genótipos de sorgo GR 11111 e TX 430 x GR 111, resistentes, GB 3B, de resistência moderada e BR 007B, suscetível ao pulgão $S$. graminum, oriundos do Banco Ativo de Germoplasma da Embrapa-Centro Nacional de Pesquisa de Milho e Sorgo. 
Os genótipos de sorgo foram cultivados em vasos com capacidade para 5,0 kg de solo e mantidos em casa de vegetação. Para iniciar a criação, os pulgões foram coletados na cultura do sorgo, na região de Jaboticabal e transferidos para as plantas aos 30 dias de idade. Cada vaso era protegido por gaiola cilíndrica de metal, revestida de tecido voile para evitar a ocorrência de parasitóides.

Adultos de $C$. sanguinea foram coletados na cultura da couve, no campus da Unesp/FCAV, Jaboticabal, SP e transferidos para gaiolas de PVC de $10 \mathrm{~cm}$ de diâmetro, revestidas internamente com papel-filtro para obtenção dos ovos, que foram transferidos, diariamente, para placas de Petri de 9,0 cm de diâmetro. Estes adultos foram alimentados diariamente com pulgão $S$. graminum criado em seções de folhas de sorgo da cultivar suscetível BR 601, para evitar qualquer pré-condicionamento alimentar do inseto.

Larvas recém-eclodidas, que já se moviam com agilidade, foram individualizadas. Segundo Hodek (1973), após a eclosão, larvas de coccinelídeos geralmente permanecem agrupadas sobre os resíduos dos ovos por períodos de até um dia, para ocorrer a esclerotização completa de seus exoesqueletos. As larvas de $C$. sanguinea foram alimentadas diariamente, ad libitum, com ninfas e adultos de S. graminum criados nas plantas de cada genótipo de sorgo. Cada parcela experimental constou de um tubo de vidro de $8,0 \mathrm{~cm}$ de altura por $2,5 \mathrm{~cm}$ de diâmetro contendo o predador e a presa. Os insetos foram mantidos à temperatura de $25 \pm 1^{\circ} \mathrm{C}$ e fotoperíodo de 12 horas. O delineamento experimental utilizado foi o inteiramente casualizado com 20 repetições. Os dados relacionados à sobrevivência foram transformados em arc sen $(\mathrm{P} / 100)^{1 / 2} \mathrm{e}$ as médias comparadas pelo teste de Tukey a 5\% de probabilidade.

Avaliou-se a duração de cada ínstar, dos períodos larval, pré-pupal, pupal e de larva a adulto e determinou-se a sobrevivência de $C$. sanguinea durante cada fase de desenvolvimento. Avaliaram-se o peso de larvas do predador durante cada ínstar, 24 horas após cada ecdise, o peso de adultos e a capacidade de oviposição diária e total durante 15 dias.

\section{Resultados e Discussão}

Apenas as durações do quarto ínstar e da fase larval e do período de larva a adulto de $C$. sanguinea foram influenciadas pelos genótipos de sorgo (Tabela 1). Geralmente, os coccinelídeos durante o primeiro, segundo e terceiro ínstares, consomem menor número de presa do que larvas de quarto ínstar. Conseqüentemente, a quantidade de pulgões $S$. graminum consumida durante estes ínstares precedentes não foi suficiente para refletir o efeito indireto dos genótipos de sorgo utilizados na criação do pulgão sobre a duração destas fases de desenvolvimento do predador. Por sua vez, a duração do quarto ínstar e da fase larval e do período de larva a adulto foi prolongada quando as larvas foram supridas com pulgões criados no genótipo resistente GR 11111 em relação àquelas alimentadas com pulgões criados nos demais genótipos.

Tabela 1. Duração e sobrevivência das fases de desenvolvimento de Cycloneda sanguinea alimentada com Schizaphis graminum criado em genótipos de sorgo resistentes (GR 11111 e TX 430 x GR 111), moderadamente resistente (GB 3B) e suscetível (BR 007B). Jaboticabal, SP, 2002(1).

\begin{tabular}{|c|c|c|c|c|c|c|c|c|}
\hline \multirow[t]{2}{*}{ Genótipos } & \multicolumn{2}{|c|}{ Primeiro ínstar } & \multicolumn{2}{|c|}{ Segundo ínstar } & \multicolumn{2}{|c|}{ Terceiro ínstar } & \multicolumn{2}{|c|}{ Quarto ínstar } \\
\hline & Duração & Sobrevivência & Duração & Sobrevivência & Duração & Sobrevivência & Duração & Sobrevivência \\
\hline GR 11111 & $1,9 \pm 0,1 \mathrm{a}$ & $95,0 \pm 5,0 \mathrm{a}$ & $1,2 \pm 0,1 \mathrm{a}$ & $100,0 \pm 0,0 \mathrm{a}$ & $1,8 \pm 0,1 \mathrm{a}$ & $100,0 \pm 0,0 \mathrm{a}$ & $2,6 \pm 0,1 \mathrm{a}$ & $100,0 \pm 0,0 \mathrm{a}$ \\
\hline TX 430 x GR 111 & $2,0 \pm 0,0 \mathrm{a}$ & $100,0 \pm 0,0 \mathrm{a}$ & $1,0 \pm 0,0 \mathrm{a}$ & $100,0 \pm 0,0 \mathrm{a}$ & $2,0 \pm 0,0 \mathrm{a}$ & $100,0 \pm 0,0 \mathrm{a}$ & $2,0 \pm 0,0 \mathrm{~b}$ & $100,0 \pm 0,0 \mathrm{a}$ \\
\hline GB 3B & $2,0 \pm 0,0 \mathrm{a}$ & $100,0 \pm 0,0 \mathrm{a}$ & $1,1 \pm 0,1 \mathrm{a}$ & $100,0 \pm 0,0 \mathrm{a}$ & $1,7 \pm 0,1 \mathrm{a}$ & $100,0 \pm 0,0 \mathrm{a}$ & $2,2 \pm 0,1 b$ & $100,0 \pm 0,0 \mathrm{a}$ \\
\hline BR 007B & $2,0 \pm 0,0 \mathrm{a}$ & $100,0 \pm 0,0 \mathrm{a}$ & $1,3 \pm 0,1 \mathrm{a}$ & $100,0 \pm 0,0 \mathrm{a}$ & $1,7 \pm 0,1 \mathrm{a}$ & $100,0 \pm 0,0 \mathrm{a}$ & $2,0 \pm 0,0 \mathrm{~b}$ & $100,0 \pm 0,0 \mathrm{a}$ \\
\hline Média & 2,0 & 99,0 & $\overline{1,1}$ & 100,0 & 1,8 & 100,0 & 2,2 & 100,0 \\
\hline \multirow[t]{3}{*}{$\mathrm{CV}(\%)$} & 5,9 & 7,5 & 29,9 & - & 21,4 & - & 14,9 & - \\
\hline & \multicolumn{2}{|c|}{ Fase larval } & \multicolumn{2}{|c|}{ Pré-pupa } & \multicolumn{2}{|r|}{ Pupa } & \multicolumn{2}{|c|}{ Larva a adulto } \\
\hline & Duração & Sobrevivência & Duração & Sobrevivência & Duração & Sobrevivência & Duração & Sobrevivência \\
\hline GR 11111 & $8,6 \pm 0,1 \mathrm{a}$ & $95,0 \pm 5,0 \mathrm{a}$ & $1,1 \pm 0,1 \mathrm{a}$ & $100,0 \pm 0,0 \mathrm{a}$ & $3,1 \pm 0,1 \mathrm{a}$ & $100,0 \pm 0,0 \mathrm{a}$ & $11,7 \pm 0,1 \mathrm{a}$ & $95,0 \pm 5,0 \mathrm{a}$ \\
\hline TX 430 x GR 111 & $8,0 \pm 0,0 \mathrm{~b}$ & $100,0 \pm 0,0 \mathrm{a}$ & $1,0 \pm 0,0 \mathrm{a}$ & $100,0 \pm 0,0 \mathrm{a}$ & $3,1 \pm 0,1 \mathrm{a}$ & $100,0 \pm 0,0 \mathrm{a}$ & $11,1 \pm 0,1 b$ & $100,0 \pm 0,0 \mathrm{a}$ \\
\hline GB 3B & $8,1 \pm 0,1 b$ & $100,0 \pm 0,0 \mathrm{a}$ & $1,0 \pm 0,0 \mathrm{a}$ & $100,0 \pm 0,0 \mathrm{a}$ & $3,1 \pm 0,1 \mathrm{a}$ & $100,0 \pm 0,0 \mathrm{a}$ & $11,1 \pm 0,1 b$ & $100,0 \pm 0,0 \mathrm{a}$ \\
\hline BR 007B & $8,1 \pm 0,1 \mathrm{~b}$ & $100,0 \pm 0,0 \mathrm{a}$ & $1,1 \pm 0,1 \mathrm{a}$ & $100,0 \pm 0,0 \mathrm{a}$ & $3,3 \pm 0,1 \mathrm{a}$ & $100,0 \pm 0,0 \mathrm{a}$ & $11,4 \pm 0,1 \mathrm{ab}$ & $100,0 \pm 0,0 \mathrm{a}$ \\
\hline Média & 10,8 & 98,7 & 1,0 & 100,0 & 3,1 & 100,0 & 11,3 & 98,7 \\
\hline CV $(\%)$ & 4,4 & 8,8 & 19,2 & - & 4,3 & - & 10,7 & 7,5 \\
\hline
\end{tabular}

(1) Médias seguidas pela mesma letra, na coluna, não diferem entre si pelo teste de Tukey a 5\% de probabilidade; os dados representam a média de 20 repetições \pm erro-padrão da média e, para análise estatística, os dados relacionados à sobrevivência foram transformados em arc sen $(\mathrm{P} / 100)^{1 / 2}$. 
O genótipo GR tem como tipos de resistência ao pulgão-verde $S$. graminum a não-preferência (Cruz \& Vendramim, 1989) e a antibiose (Cruz \& Vendramim, 1995). Características da planta, como teor de aleloquímicos, nutrição, morfologia e fenologia que conferem resistência a insetos-praga podem atuar adversamente sobre os insetos benéficos, caracterizando a resistência do tipo antibiose (Heinz \& Zalom, 1996). No presente trabalho, a antibiose da cultivar GR 11111 afetou indiretamente o desenvolvimento do inimigo natural, $C$. sanguinea, concordando com Vendramim (2002), que mencionou que o aleloquímico produzido pela planta hospedeira afeta negativamente a praga, mas esta sobrevive, mantendo em seu organismo certa concentração da substância química, que afetará o desenvolvimento dos inimigos naturais.

Rice \& Wilde (1989) constataram também que genótipos de sorgo com mecanismo de antibiose ao pulgão S. graminum prolongaram a fase larval de H. convergens. Segundo Botrell et al. (1998), a fonte de alimentação do inseto fitófago irá afetar a nutrição, tamanho, sobrevivência, período de desenvolvimento e densidade na cultura do inseto-praga mas também do inimigo natural.

Apesar do genótipo GR 11111 ter provocado indiretamente o prolongamento na fase larval de C. sanguinea, este predador apresentou alto porcentual de sobrevivência, tanto na fase larval quanto no período de larva a adulto, quando alimentado com pulgões criados em folhas do referido genótipo resistente, não diferindo significativamente das larvas alimentadas com os pulgões criados nos demais genótipos (Tabela 1). Embora os resultados indiquem que GR 11111 não afetou negativamente os aspectos biológicos de $C$. sanguinea, Figueira et al. (2002), avaliando os efeitos destes mesmos genótipos de sorgo no desenvolvimento do crisopídeo C. externa, alimentado com $S$. graminum, constatou influência negativa do GR 11111 na duração e sobrevivência da fase de pré-pupa, peso de larvas de terceiro ínstar e do adulto e na longevidade de fêmeas. Segundo Giles et al. (2001), os fatores que influenciam as interações tritróficas são variáveis entre as espécies afidófagas.

Os períodos pré-pupal e pupal, de $C$. sanguinea, foram em média de 1,0 e 3,1 dias, respectivamente, com porcentual de sobrevivência de $100,0 \%$ (Tabela 1), e similares aos detectados por Santos \& Pinto (1981) e Santa-Cecília et al. (2001) para larvas deste predador alimentadas, respectivamente, com pulgões Toxoptera aurantii (Boyer de Fonscolombe) e com S. graminum.

O peso de larvas de $C$. sanguinea de segundo ínstar, cuja média foi de $1,7 \mathrm{mg}$, não refletiu o efeito dos genótipos de sorgo nos quais foi criada a sua presa, S. graminum (Tabela 2). No entanto, nos demais ínstares e fase adulta, esta variável foi diferenciada em razão do genótipo. Larvas de terceiro ínstar supridas com $S$. graminum criado em folhas dos genótipos BR 007B e TX 430 x GR 111, respectivamente, suscetível e resistente a este afídeo, atingiram menor peso do que as alimentadas com pulgões criados no genótipo GB 3B, de resistência moderada, mas não diferiram em peso das larvas relacionadas ao tratamento GR 11111, também resistente.

Genótipos de sorgo de moderada resistência (GB 3B) e suscetível (BR 007B) ao pulgão proporcionaram maior peso às larvas de quarto ínstar de C. sanguinea (Tabela 2). Situação similar ocorreu com os adultos, que atingiram maior peso quando foram alimentados com pulgões criados no genótipo suscetível (BR 007B), em comparação ao peso de adultos que foram supridos com pulgões mantidos nos genótipos resistentes TX 430 x GR 111 e GR 11111.

Esses resultados evidenciam uma influência negativa dos genótipos resistentes sobre o predador. De acordo com Giles et al. (2001), o tamanho de

Tabela 2. Peso de larvas e adultos de Cycloneda sanguinea alimentados com Schizaphis graminum, criado em genótipos de sorgo resistentes (GR 11111 e TX 430 x GR 111), moderadamente resistente (GB 3B) e suscetível (BR 007B). Jaboticabal, SP, $2002^{(1)}$.

\begin{tabular}{lcccc}
\hline Genótipos & \multicolumn{4}{c}{ Peso (mg) } \\
\cline { 2 - 5 } & $\begin{array}{c}\text { Segundo } \\
\text { ínstar }\end{array}$ & $\begin{array}{c}\text { Terceiro } \\
\text { ínstar }\end{array}$ & $\begin{array}{c}\text { Quarto } \\
\text { ínstar }\end{array}$ & Adulto \\
\hline GR 11111 & $1,6 \pm 0,08 \mathrm{a}$ & $3,6 \pm 0,18 \mathrm{ab}$ & $9,3 \pm 0,4 \mathrm{~b}$ & $12,0 \pm 0,39 \mathrm{c}$ \\
TX 430 x GR 111 & $1,6 \pm 0,08 \mathrm{a}$ & $3,4 \pm 0,10 \mathrm{~b}$ & $10,4 \pm 0,13 \mathrm{~b}$ & $13,0 \pm 0,39 \mathrm{bc}$ \\
GB 3B & $1,8 \pm 0,06 \mathrm{a}$ & $4,4 \pm 0,24 \mathrm{a}$ & $16,9 \pm 0,59 \mathrm{a}$ & $14,1 \pm 0,51 \mathrm{ab}$ \\
BR 007B & $1,6 \pm 0,11 \mathrm{a}$ & $3,4 \pm 0,27 \mathrm{~b}$ & $15,0 \pm 0,80 \mathrm{a}$ & $15,7 \pm 0,36 \mathrm{a}$ \\
\hline Média & 1,7 & 3,7 & 12,9 & 13,7 \\
CV (\%) & 14,9 & 19,8 & 12,8 & 10,2 \\
\hline (1)Médias seguidas pela mesma letra, na coluna, não diferem entre si pelo \\
teste de Tukey a 5\% de probabilidade; os dados representam a média de \\
20 repetições \pm erro-padrão da média.
\end{tabular}


coccinelídeos adultos pode influenciar significativamente as populações subseqüentes, ocasionando redução de suas fecundidades. No entanto, este efeito não foi detectado no presente trabalho, uma vez que o número diário e total de ovos colocados por $C$. sanguinea não diferiu significativamente quando fêmeas foram alimentadas com $S$. graminum criado nos genótipos de sorgo resistentes e suscetíveis a este afídeo (Tabela 3). O fato de a fecundidade das fêmeas não ter sido afetada negativamente pelos genótipos resistentes coincide com os resultados obtidos por Figueira et al. (2002), para C. externa, cujas larvas foram alimentadas com $S$. graminum criado nos mesmos genótipos de sorgo, do presente trabalho.

Os genótipos resistentes GR 11111 e TX 430 x GR 111 não afetaram adversamente o desenvolvimento e a fecundidade de $C$. sanguinea, sendo a resistência destes genótipos compatível com esta espécie de predador. Cruz \& Vendramim (1995), porém, constataram que estes genótipos resistentes afetam a biologia do pulgão $S$. graminum, alterando o seu crescimento populacional, prolongando o seu desenvolvimento e reduzindo o seu peso. Segundo Messina \& Sorenson (2001), este efeito, característico da resistência do tipo antibiose, ocasiona indiretamente o aumento da razão predador/presa e, por conseguinte, evita que a população do pulgão atinja maiores densidades, em que o inimigo natural tornese ineficiente.

Tabela 3. Número diário e total de ovos colocados durante 15 dias por Cycloneda sanguinea alimentada com Schizaphis graminum criado em genótipos de sorgo resistentes (GR 11111 e TX 430 x GR 111), moderadamente resistente (GB 3B) e suscetível (BR 007B). Jaboticabal, SP, $2002^{(1)}$.

\begin{tabular}{lcc}
\hline Genótipos & \multicolumn{2}{c}{ Oviposição } \\
\cline { 2 - 3 } & Diária & Total \\
\hline GR 11111 & $20,2 \pm 2,44 \mathrm{a}$ & $199,8 \pm 43,53 \mathrm{a}$ \\
TX 430 x GR 111 & $19,0 \pm 2,20 \mathrm{a}$ & $191,8 \pm 45,45 \mathrm{a}$ \\
GB 3B & $22,0 \pm 1,70 \mathrm{a}$ & $257,8 \pm 18,26 \mathrm{a}$ \\
BR 007B & $18,1 \pm 1,59 \mathrm{a}$ & $187,2 \pm 13,63 \mathrm{a}$ \\
\hline Média & 19,8 & 209,1 \\
CV $(\%)$ & 22,8 & 35,8 \\
\hline
\end{tabular}

(1)Médias seguidas pela mesma letra, na coluna, não diferem entre si pelo teste de Tukey a 5\% de probabilidade; os dados representam a média de 20 repetições \pm erro-padrão da média.

\section{Conclusões}

1. A alimentação do pulgão $S$. graminum com os genótipos resistentes de sorgo, GR 11111 e TX 430 x GR 111, não exerce efeito adverso no desenvolvimento e fecundidade do seu predador $C$. sanguinea, durante uma geração.

2. Os genótipos GR 11111 e TX 430 x GR 111 são compatíveis com o predador C. sanguinea, tornando viável a utilização integrada de plantas resistentes e controle biológico efetuado por este predador no manejo integrado do pulgão $S$. graminum na cultura do sorgo.

\section{Referências}

BOTRELL, D. G.; BARBOSA, P.; GOULD, F. Manipulating natural enemies by plant variety selection and modification: a realistic strategy? Annual Review of Entomology, Palo Alto, v. 43, p. 347-367, 1998.

CRUZ, I.; VENDRAMIM, J. D. Efeito da alternância de genótipos de sorgo resistente e suscetível na biologia de Schizaphis graminum (Rond.) (Homoptera: Aphididae). Anais da Sociedade Entomológica do Brasil, Londrina, v. 27, n. 2, p. 281-287, 1998.

CRUZ, I.; VENDRAMIM, J. D. Efeito de diferentes genótipos de sorgo resistentes no desempenho do pulgão-verde Schizaphis graminum Rond. Anais da Sociedade Entomológica do Brasil, Piracicaba, v. 24, n. 2, p. 253-263, 1995.

CRUZ, I.; VENDRAMIM, J. D. Não-preferência como mecanismo de resistência de sorgo ao pulgão-verde. Pesquisa Agropecuária Brasileira, Brasília, v. 24, n. 3, p. 329-335, mar. 1989.

FARID, A.; JOHNSON, J. B.; QUISENBERRY, S. S. Compatibility of a coccinellid predator with a Russian wheat aphid resistant wheat. Journal of the Kansas Entomological Society, Lawrence, v. 70, n. 2, p. 114-119, 1997.

FIGUEIRA, L. K.; LARA, F. M.; CRUZ, I. Efeito de genótipos de sorgo sobre o predador Chrysoperla externa (Hagen) (Neuroptera: Chrysopidae) alimentado com Schizaphis graminum (Rondani) (Hemiptera: Aphididae). Neotropical Entomology, Londrina, v. 31, n. 1, p. 133-139, 2002. 
GILES, K. L.; BERBERET, R. C.; ZARRABI, A. A.; DILLWITH, J. W. Influence of alfalfa cultivar on suitability of Acyrthosiphon kondoi (Homoptera: Aphididae) for survival and development of Hippodamia convergens and Coccinella septempunctata (Coleoptera: Coccinellidae). Journal of Economic Entomology, Lanham, v. 95, n. 3, p. $552-557,2002$.

GILES, K. L.; STOCKLAND, R.; MADDEN, R. D.; PAYTON, M. E.; DILLWITH, J. W. Preimaginal survival and development of Coleomegilla maculata and Hippodamia convergens (Coleoptera: Coccinellidae) reared on Acyrthosiphon pisum: effects of host plants. Environmental Entomology, Lanham, v. 30, n. 5, p. 961-971, 2001.

HEINZ, K. M.; ZALOM, F. G. Performance of the predator Delphastus pusillus on Bemisia resistant and susceptible tomato lines. Entomologia Experimentalis et Applicata, Dordrecht, v. 81, n. 3, p. 345-352, 1996.

HODEK, I. Life history and biological properties. In: HODEK, I. Biology of Coccinellidae. The Hague: W. Junk N. V., 1973. p. 70-75.

IPERTI, G. Biodiversity of predaceous Coccinellidae in relation to bioindication and economic importance. Agriculture, Ecosystems and Environment, Amsterdam, v. 74, n. 1/3, p. 323-342, 1999.

ISENHOUR, D. J.; WISEMAN, B. R.; LAYTON, R. C. Enhanced predation by Orius insidiosus (Hemiptera: Anthocoridae) on larvae of Heliothis zea and Spodoptera frugiperda (Lepidoptera: Noctuidae) caused by prey feeding on resistant genotypes. Environmental Entomology, Lanham, v. 18, n. 3, p. 418-422, 1989.

LARA, F. M. Tipos de resistência. In: LARA, F. M. Princípios de resistência de plantas a insetos. 2. ed. São Paulo: Ícone, 1991. p. 35-74.

MESSINA, F. J.; SORENSON, S. M. Effectiveness of lacewing larvae in reducing Russian wheat aphid populations on susceptible and resistant wheat. Biological Control, Orlando, v. 21, n. 1, p. 19-26, 2001.

OBRYCKI, J. J.; KRING, T. J. Predaceous Coccinellidae in biological control. Annual Review of Entomology, Palo Alto, v. 43, p. 295-321, 1998

RICE, M. E.; WILDE, G. E. Antibiosis effect of sorghum on the convergent lady beetle (Coleoptera: Coccinellidae), a third-trophic level predator of the greenbug (Homoptera: Aphididae). Journal of Economic Entomology, Lanham, v. 82 , n. 2 , p. $570-573,1989$.

SANTA-CECÍLIA, L. V. C.; GONÇALVES-GERVÁSIO, R. C. R.; TÔRRES, R. M. S.; NASCIMENTO, F. R. Aspectos biológicos e consumo alimentar de larvas de Cycloneda sanguinea (Linnaeus, 1763) (Coleoptera: Coccinellidae) alimentadas com Schizaphis graminum (Rondani, 1852) (Hemiptera: Aphididae). Ciência e Agrotecnologia, Lavras, v. 25, n. 6, p. 1273-1278, 2001.

SANTOS, G. P.; PINTO, A. C. Q. Biologia de Cycloneda sanguinea e sua associação com pulgão em mudas de mangueira. Pesquisa Agropecuária Brasileira, Brasília, v. 16, n. 4, p. 473-476, abr. 1981

SILVA, E. A. da; LARA, F. M. Influência de genótipos de batata, Solanum spp., na predação de Myzus persicae (Sulz.) (Hemiptera: Aphididae) por Cycloneda sanguinea (L.) (Coleoptera: Coccinellidae). Revista Ecossistema, Espírito Santo do Pinhal, v. 24, p. 109-112, 1999.

VENDRAMIM, J. D. O controle biológico e a resistência de plantas. In: PARRA, J. R. P.; BOTELHO, P. S. M.; CORRÊA-FERREIRA, B. S.; BENTO, J. M. S. (Ed.). Controle biológico no Brasil: parasitóides e predadores. São Paulo: Manole, 2002. p. 511-519.

VERKERK, R. H. J.; LEATHER, S. R.; WRIGHT, D. J. The potential for manipulating crop-pest-natural enemy interactions for improved insect pest management. Bulletin of Entomological Research, Wallingford, v. 88, n. 5, p. 493-501, 1998. 